\title{
Effective microscopic models for sympathetic cooling of atomic gases
}

\author{
Roberto Onofrio ${ }^{1,2}$ and Bala Sundaram ${ }^{3}$ \\ ${ }^{1}$ Dipartimento di Fisica e Astronomia "Galileo Galilei", Università di Padova, Via Marzolo 8, Padova 35131, Italy \\ ${ }^{2}$ Department of Physics and Astronomy, Dartmouth College, 6127 Wilder Laboratory, Hanover, NH 03755, USA \\ ${ }^{3}$ Department of Physics, University of Massachusetts, Boston, MA 02125, USA
}

\begin{abstract}
Thermalization of a system in the presence of a heat bath has been the subject of many theoretical investigations especially in the framework of solid state physics. In this setting, the presence of a large bandwidth for the frequency distribution of the harmonic oscillators schematizing the heat bath is crucial, as emphasized in the Caldeira-Leggett model. By contrast, ultracold gases in atomic traps oscillate at well-defined frequencies and therefore seem to lie outside the Caldeira-Leggett paradigm. We introduce interaction Hamiltonians which allow us to adapt the model to an atomic physics framework. The intrinsic nonlinearity of these models differentiates them from the original Caldeira-Leggett model and calls for a nontrivial stability analysis to determine effective ranges for the model parameters. These models allow for molecular dynamics simulations of mixtures of ultracold gases, which is of current relevance for optimizing sympathetic cooling in degenerate Bose-Fermi mixtures.
\end{abstract}

PACS numbers: 37.10.De, 02.70.Ns, 67.85.Pq, 05.70.Ln

\section{INTRODUCTION}

The study of dynamical systems interacting with an external environment plays an essential role in classical and quantum physics, since long-term properties of any system are going to be affected by the outside world unless complete adiabaticity can be assumed at all times. In particular, the presence of environments having the energy of their constituent particles shared according to the Boltzmann distribution, also named 'baths', is at the basis of the canonical ensemble approach in equilibrium statistical mechanics, with its large variety of physical implications. A microscopic analysis of the system-bath interplay is possible by writing explicitly the bath degrees of freedom and integrating out their effect on the target particle. Along these lines, various authors have determined sufficient conditions under which a Langevin dynamics, and the consequent thermalization, holds. This has been achieved by considering a bath made of non-interacting harmonic oscillators, which interact linearly with the target particle, the so-called Caldeira-Leggett model [1,4].

More recently, studies have focused attention on baths endowed with finite resources, such as a finite number of harmonic oscillators distributed in a finite bandwidth, i.e. allowing for a non-zero infrared cut-off and a finite ultraviolet cut-off [5 [10]. In particular, the dynamics of a target harmonic oscillator interacting via a linear and translationally invariant term with a bath, composed of a finite number of harmonic oscillators whose frequencies are all in a limited range, was studied in detail. The principal outcome of this analysis, presented in [5], can be summarized as follows. To ensure thermalization, the frequency of the test particle must lie within the band of bath frequencies, which in turn must also have a large enough bandwidth. In the absence of any one of these conditions the test particle does not thermalize to the temperature of the bath. The implication of this is either that the energy distribution is not amenable to a Boltzmann distribution or that the resulting effective temperature of the test particle is significantly different from that of the bath.

Thermalization plays a crucial role in sympathetic cooling of atomic species trapped in magnetic or optical potentials. This was first demonstrated experimentally when ${ }^{85} \mathrm{Rb}$, a bosonic Rubidium isotope which is hard to cool using evaporating techniques, was successfully cooled via thermal contact with ${ }^{87} \mathrm{Rb}$ [11]. The technique is even more crucial in the case of fermionic atomic species where direct evaporative cooling loses its efficiency in the degenerate regime due to fundamental limitations arising from the Pauli principle [12 14]. A number of studies have led to predictions for new phase transitions occurring for ultracold fermions in single traps or optical lattices, and the current experimental focus is on achieving temperatures low enough to observe these effects [15].

The Caldeira-Leggett model, in its original form, cannot be used to describe thermalization in the atomic physics framework. In its applications to solid-state systems, this model is meaningful due to the multiplicity of normal modes associated with lattice vibrations and the need for a continuous density of states, as in the Debye model [16]. In contrast, in atom traps no phonon-mediated interactions exist in principle either in single traps or in optical lattices. Thus coolant and target species both have single values for the trapping frequencies which means that, based on the Caldeira-Leggett model, no thermalization is expected. This is further heightened in those proposals where the two species are deliberately required to have significantly different trapping frequencies in order to optimize sympathetic cooling [17 23]. Of course, this inference is in stark contrast to the experimentally observed success of sympathetic cooling in a range of atomic mixtures. 
In this paper, we demonstrate that it is possible to choose bath-test particle interactions which reconcile the Caldeira-Leggett model with the experimentally manifest effectiveness of sympathetic cooling. The details of the dynamics of thermalization is studied as well in terms of the stability with respect to different choices of the model parameters. The model we discuss is purely classical, both in regard to the dynamical evolution and to the choice of Boltzmann heat baths. While classical dynamics is generally consider sufficient to describe the motion of ultracold atoms under current experimental conditions, in the quantum degenerate regime the Boltzmann energy distribution should be superseded by Bose or Fermi distributions. This work should be therefore considered as a prelude, in the Dulong-Petit classical limit, to the analysis of fully degenerate ultracold quantum gases, setting the stage and the language for future work aimed at a more comprehensive analysis of dynamical situations such as the sympathetic cooling of Bose-Fermi mixtures.

The paper is organized as follows. In Section II we introduce two interaction Hamiltonians differing in their selectivity with respect to the relative velocity of the interacting particles, and we make explicit the Hamilton's equations and associated considerations. In Section III we discuss an interesting phenomenon which occurs in the strong coupling limit of repulsive interaction, i.e. the fact that the test particle experiences an effective double-well potential which influences the dynamics of cooling. This is demonstrated both through numerical and approximate fixed point analyses, which then results in a phase diagram delineating various regimes in the parameter space. Section IV presents the results of numerical simulations showing the approach to thermal equilibrium and the relaxation dynamics in terms of the interaction energy. In the process, we revisit some assumptions implicit in discussions of thermalization, which are not necessarily satisfied in models like ours. In the conclusions, we discuss the relevance of this model to actual simulations of ultracold atomic mixtures, as well as several directions for expanding the applicability of our models. A particular point of interest is the possible application of recent results on shortcuts to adiabaticity for hastening the rate of thermalization in our dynamical models.

\section{INTERACTION HAMILTONIANS}

The classical Hamiltonian for a 1D harmonic oscillator of mass $M$ and angular frequency $\Omega$, which we will refer to as a 'test particle', in the presence of a bath made of $N_{b}$ harmonic oscillators of mass $m$ and generic frequency $\omega_{n} \in\left[\omega_{I R}, \omega_{U V}\right]$ linearly coupled to it, is written as

$$
H_{t o t}=\frac{P^{2}}{2 M}+\frac{1}{2} M \Omega^{2} Q^{2}+\sum_{n=1}^{N_{b}}\left[\frac{p_{n}^{2}}{2 m}+\frac{1}{2} m \omega_{n}^{2}\left(q_{n}-Q\right)^{2}\right] .
$$

The associated Hamilton equations are

$$
\begin{aligned}
\dot{Q} & =\frac{P}{M}, \\
\dot{P} & =-M \Omega^{2} Q+\sum_{n=1}^{N_{b}} m \omega_{n}^{2}\left(q_{n}-Q\right), \\
\dot{q_{n}} & =\frac{p_{n}}{m} \\
\dot{p_{n}} & =-m \omega_{n}^{2}\left(q_{n}-Q\right) .
\end{aligned}
$$

These show that the effect of the bath on the test particle leads to a modified 'spring constant' (which can also be viewed as a combined change in mass and frequency) such that $M \Omega^{2} \mapsto M \Omega^{2}+m \sum_{n=1}^{N_{b}} \omega_{n}^{2}$, and in an effective force equal to $-m \sum_{n=1}^{N_{b}} \omega_{n}^{2} q_{n}$, where the latter depends on the bath dynamics. If the bath oscillators all have the same frequency then the driving force is simply proportional to $\sum_{n} q_{n}$, which averages to zero for a large number of bath oscillators with uniform distribution of initial conditions in phase space. This precludes any exchange of net energy between the bath and the test particle. This implies that thermalization relies on and requires the existence of a large number of distinct frequencies for the bath oscillators. As such, atoms oscillating in a harmonic trap with a common angular frequency, $\omega$, do not fall within the Caldeira-Leggett framework. To address thermalization in this specific situation, the interaction between the test particle and the bath particles needs to be modified, and we will consider a Hamiltonian of the generic form

$$
H_{t o t}=\frac{P^{2}}{2 M}+\frac{1}{2} M \Omega^{2} Q^{2}+\sum_{n=1}^{N_{b}}\left(\frac{p_{n}^{2}}{2 m}+\frac{1}{2} m \omega^{2} q_{n}^{2}\right)+H_{\mathrm{int}}\left(Q, P, q_{n}, p_{n}\right)
$$


Given our motivation, the first distinctive requirement in $H_{\text {int }}$ is that interactions between atoms be highly localized in configuration space, being described either by pseudopotentials with zero range or by finite range (dipolar) interactions. Locality of the bath-test particle interaction, first introduced to our knowledge in the context of a microscopic description of a measurement apparatus [24], is easily achieved by introducing a spatial 'filter', for instance of a Gaussian nature. This implies an interaction Hamiltonian of the generic form

$$
H_{i n t}\left(Q, q_{n}, P, p_{n}\right)=\gamma \sum_{n=1}^{N_{b}} f\left(q_{n}-Q, v_{n}-V\right) \exp \left[-\frac{\left(q_{n}-Q\right)^{2}}{\lambda^{2}}\right]
$$

Thus the test particle-bath interaction Hamiltonian is dependent on two parameters, the coupling strength $\gamma$ and the range $\lambda$. The interaction Hamiltonian is basically negligible if $\left|q_{n}-Q\right|>>\lambda$. The generic function $f$ is chosen to fulfill Galilean invariance, as reflected in the explicit dependence on the differences between the coordinates and velocities of the involved particles, with $V=P / M$ and $v_{n}=p_{n} / m$. For the remainder of our analysis, we will contrast two forms of the function $f$. The first is where $f$ is constant, which is a velocity-independent situation, while the second choice takes $f$ to be a quadratic function of the relative velocity. Having in mind applications in the atomic physics arena, the coupling strength $\gamma$ should eventually be related to the scattering length in the case of pseudopotentials. Examples of these are the zero-range approximation usually adopted in the Gross-Pitaevskii equation for Bose gases (in which case $\lambda$ is chosen to be zero), or to the van der Waals potential in the case of finite-range interactions as in dipolar gases.

\section{A. Velocity-independent interaction Hamiltonian}

Of the various possibilities available for the function $f$, the simplest is a constant value, that is

$$
H_{\text {int }}\left(Q, q_{n}\right)=\gamma_{E} \sum_{n=1}^{N_{b}} \exp \left[-\frac{\left(q_{n}-Q\right)^{2}}{\lambda^{2}}\right]
$$

Here a significant impulsive force occurs at each interaction between the test particle and a particle of the bath if they are within a distance of order of $\lambda$ of each other. The strength of the interaction is controlled by the parameter $\gamma_{E}$ which has the dimensions of energy and sets the maximal possible energy exchange during the interaction, which is achieved in the limit $\lambda \rightarrow 0$, a sort of force 'kick' of constant amplitude. The sign of $\gamma_{E}$ determines the attractive $\left(\gamma_{E}<0\right)$ or repulsive $\left(\gamma_{E}>0\right)$ character of the interaction, as in the former case the interaction energy is minimized at the smallest distances, and this trend is reversed in the latter situation.

The corresponding Hamilton equations of motion are

$$
\begin{aligned}
\dot{Q} & =\frac{P}{M} \\
\dot{P} & =-M \Omega^{2} Q-2 \frac{\gamma_{E}}{\lambda^{2}} \sum_{n=1}^{N_{b}} \exp \left[-\frac{\left(q_{n}-Q\right)^{2}}{\lambda^{2}}\right]\left(q_{n}-Q\right), \\
\dot{q}_{n} & =\frac{p_{n}}{m} \\
\dot{p}_{n} & =-m \omega^{2} q_{n}+2 \frac{\gamma_{E}}{\lambda^{2}} \exp \left[-\frac{\left(q_{n}-Q\right)^{2}}{\lambda^{2}}\right]\left(q_{n}-Q\right) .
\end{aligned}
$$

In the limit of large $\lambda$ the total Hamiltonian resembles Eq. (1) with the uniform bath frequency Caldeira-Leggett interaction, but with important differences at large $\gamma$ since, by expanding the exponential function to second order in $q_{n}-Q$

$$
\exp \left[-\left(q_{n}-Q\right)^{2} / \lambda^{2}\right] \approx 1-\left(q_{n}-Q\right)^{2} / \lambda^{2}
$$

we get, aside from an irrelevant constant $\gamma_{E} N_{b}$, the following Hamiltonian

$$
H_{t o t} \simeq \frac{P^{2}}{2 M}+\frac{1}{2} M \Omega^{2}\left(1-\frac{2 \gamma_{E} N_{b}}{M \Omega^{2} \lambda^{2}}\right) Q^{2}+\sum_{n=1}^{N_{b}}\left[\frac{p_{n}^{2}}{2 m}+\frac{1}{2} m \omega^{2}\left(1-\frac{2 \gamma_{E}}{m \omega^{2} \lambda^{2}}\right) q_{n}^{2}\right]+\frac{2 \gamma}{\lambda^{2}} Q \sum_{n=1}^{N_{b}} q_{n}
$$


This shows that both test- and bath- particle frequencies are renormalized as $\Omega \rightarrow \Omega\left[1-2 \gamma_{E} N_{b} /\left(M \Omega^{2} \lambda^{2}\right)\right]^{1 / 2}$, and $\omega \rightarrow \omega\left[1-2 \gamma_{E} /\left(m \omega^{2} \lambda^{2}\right)\right]^{1 / 2}$. Additionally, there is the appearance of an external driving force, proportional to the sum of all coordinates of the proximal bath particles, which vanishes in the large, uniformly distributed, $N_{b}$ limit. Notice that, even if the bath particles and the test particle are initially chosen with degenerate frequencies and same masses, their interaction produces a relative frequency shift. Also, for comparable parameters $M \simeq m, \Omega \simeq \omega$, the harmonic trap becomes unstable when $\gamma_{E}>\bar{\gamma}_{E}=M \Omega^{2} \lambda^{2} /\left(2 N_{b}\right)$, beyond which the local effective potential seen by the test particle near the origin is that of an inverted harmonic oscillator. The overall effective potential then morphs into an (attractive) quartic potential at larger values of $Q$, as shown by further expanding the right hand side of Eq. (10) to fourth order in $q_{n}-Q$. Therefore the effective dynamics for the repulsive $\gamma_{E}>0$ case is one of a double-well for $0<\bar{\gamma}_{E}<\gamma_{E}$. No such instability occurs around the origin for negative values of $\gamma_{E}$, corresponding to attractive interactions.

In the opposite limit of small $\lambda$, at any given time the bath decouples into a subsystem of $N_{\lambda}<N_{b}$ interacting oscillators, which are within range $\lambda$ of the test particle, while the remaining $\left(N_{b}-N_{\lambda}\right)$ bath particles are irrelevant to the dynamics. The average number of oscillators $N_{\lambda}$ of the heat bath interacting at any given time with the test particle may be estimated in a stationary regime once we evaluate the oscillation amplitude of the generic oscillator $\left\langle q_{n}^{2}\right\rangle^{1 / 2}=\left(2\left\langle E_{n}\right\rangle / m \omega^{2}\right)^{1 / 2}$ where $\left\langle E_{n}\right\rangle$ is the total energy of the $n^{t h}$ particle. The equipartition theorem provides the estimate $E_{n}=K_{B} T_{b}$, so that average oscillation amplitude of a generic particle of the heat bath is $\left\langle q_{n}^{2}\right\rangle^{1 / 2}=\left(2 K_{B} T_{b} /\left(m \omega^{2}\right)\right)^{1 / 2}$, and its motion will span an interval of coordinate values $\left[-\left\langle q_{n}^{2}\right\rangle^{1 / 2},\left\langle q_{n}^{2}\right\rangle^{1 / 2}\right]$, while the test particle has a interaction range of $2 \lambda$. Considering uniform distributions over the ranges, this suggests that the number of oscillators $N_{\lambda} \simeq 2 \lambda N_{b} /\left\langle q_{n}^{2}\right\rangle^{1 / 2}$, meaning an inverse dependence on the temperature of the heat bath.

\section{B. Velocity-dependent interaction Hamiltonian}

The form assumed above for the Hamiltonian is the simplest one possible and we can, as more appropriate for collisional interactions, incorporate a dependence on the velocities of the test particle and each particle in the heat bath. We continue to assume, as in the previous case, a local spatial interaction and the interaction Hamiltonian is now given by

$$
H_{i n t}\left(Q, P, q_{n}, p_{n}\right)=\gamma_{M} \sum_{n=1}^{N_{b}}\left(\frac{p_{n}}{m}-\frac{P}{M}\right)^{2} \exp \left[-\frac{\left(q_{n}-Q\right)^{2}}{\lambda^{2}}\right]
$$

This velocity-selective Hamiltonian now corresponds to a force which depends on the velocity mismatch. Our choice is reminiscent of the fact that the elastic scattering rate between atoms is proportional to the relative velocity between the colliding particles, provided they are in close proximity to feel deviations from the original trajectory through van der Waals like forces. In this case the interaction strength is dependent upon a parameter $\gamma_{M}$ which has dimensions of mass.

The corresponding Hamilton equations are somewhat more complex than earlier due to the velocity-dependent term

$$
\begin{aligned}
\dot{Q} & =\frac{P}{M}-2 \frac{\gamma_{M}}{M} \sum_{n=1}^{N_{b}}\left(\frac{p_{n}}{m}-\frac{P}{M}\right) \exp \left[-\frac{\left(q_{n}-Q\right)^{2}}{\lambda^{2}}\right] \\
\dot{P} & =-M \Omega^{2} Q-2 \frac{\gamma_{M}}{\lambda^{2}} \sum_{n=1}^{N_{b}}\left(\frac{p_{n}}{m}-\frac{P}{M}\right)^{2} \exp \left[-\frac{\left(q_{n}-Q\right)^{2}}{\lambda^{2}}\right]\left(q_{n}-Q\right), \\
\dot{q_{n}} & =\frac{p_{n}}{m}+2 \frac{\gamma_{M}}{M}\left(\frac{p_{n}}{m}-\frac{P}{M}\right) \exp \left[-\frac{\left(q_{n}-Q\right)^{2}}{\lambda^{2}}\right], \\
\dot{p_{n}} & =-m \omega^{2} q_{n}+2 \frac{\gamma_{M}}{\lambda^{2}}\left(\frac{p_{n}}{m}-\frac{P}{M}\right)^{2} \exp \left[-\frac{\left(q_{n}-Q\right)^{2}}{\lambda^{2}}\right]\left(q_{n}-Q\right) .
\end{aligned}
$$

The main distinctive feature of this set of equations is the presence of time-dependent masses for both the target particle and the bath particles, which in a coarse graining of the dynamics correspond to time-averaged, effective renormalized masses. This feature makes a stability analysis of the solutions more delicate, requiring a fixed point analysis as detailed in the next section. In terms of the sign of the interaction, $\gamma_{M}>0$ corresponds to repulsive interactions in which minimization of the interaction energy occurs, as in the former case, at large particle separation and minimum relative velocity between the two particles. Here, instabilities occur in the attractive case of $\gamma_{M}<0$, as the interaction energy is minimized for small distances between the particles and large velocity separation. 

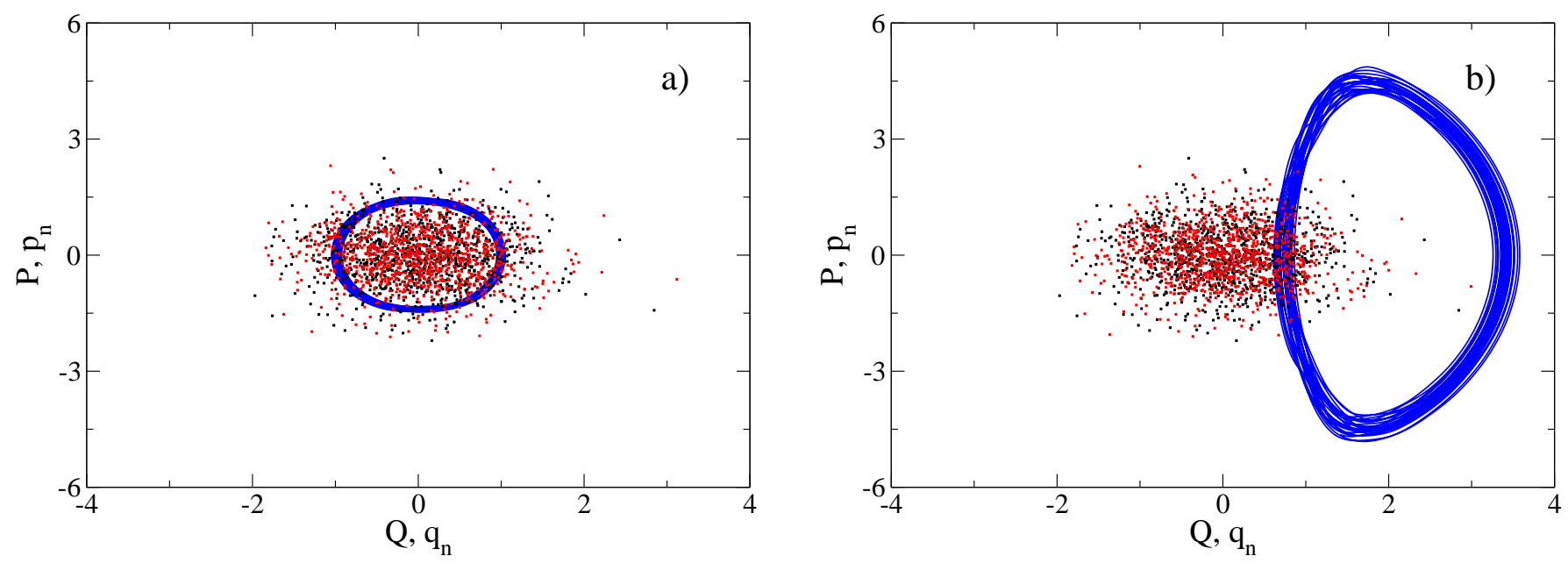

FIG. 1: (Color online) Demonstration of the transition in the dynamics from (a) a single fixed point at $(0,0)$ to $(b)$ ones off-center on changing the parameters of the velocity independent interaction. In each of the graphs, the initial (black points) and final (red) phase space locations of the $N_{b}$ bath oscillators are shown as well the blue trajectory of a single test particle. Note that the initial condition, chosen here at random, determines the shape of the trajectory in the more complicated case (b). Both cases are evaluated for $\lambda=0.25$, one test particle, $N_{b}=10^{3}, T_{b}=0.5, T_{p}=0.1, M=m=1, \omega_{n}=1$, and $\Omega=144 / 89$. (a) $\gamma_{E}=2 \times 10^{-3}$; (b) $\gamma_{E}=0.1$, with temperatures, masses, angular frequencies, parameters $\gamma_{E}, \lambda$ expressed in arbitrary units.

Although the total energy of the overall system is conserved, the presence, on the righthand side of Eqs. $(13,14)$, of forces proportional to the square of the velocity difference resembles dissipation-like behavior. This should be expected to make the energy exchange between the test particles and the bath particles occur on shorter timescales when contrasted with the velocity-independent case where the dissipative feature is lacking. We note here that a more generic interaction Hamiltonian of the form

$$
H_{\text {int }}\left(Q, P, q_{n}, p_{n}\right)=\gamma \sum_{n=1}^{N_{b}} \exp \left[\frac{\left(p_{n} / m-P / M\right)^{2}}{\nu^{2}}-\frac{\left(q_{n}-Q\right)^{2}}{\lambda^{2}}\right] .
$$

can be written where $\nu$ is a parameter related to the velocity spread in the particle ensembles. A Taylor expansion of the velocity-dependent exponential term generates the velocity-independent and velocity-dependent interactions respectively at the zeroth and second order in the parameter $\left(p_{n} / m-P / M\right) / \nu$, with the identifications of $\gamma_{E}=\gamma$ and $\gamma_{M}=\gamma / \nu^{2}$.

In discussing both types of interactions, what is shown is for a single target particle interacting with a set of bath oscillators. These relations can be readily extended to the more general situation of $N_{p}$ test particle oscillators interacting with $N_{b}$ oscillators of the heat bath, as in the subsequent simulations described in Section IV. The advantage of this more general situation is that the effective temperature of the test particles can be obtained at any time by looking at the energy distribution of the test particle ensemble, rather than taking a finite sequence of energies in a given time interval, a procedure followed in [5]. This allows for a more accurate tracking of the time evolution of the temperature, and therefore of the expected thermalization dynamics. Besides, the interaction of two clouds of particles is more appropriate to sympathetic cooling which is an important motivation for this work.

\section{LIMITS IN THE PARAMETER SPACE AND FIXED POINT ANALYSIS}

The analysis thus far, for both forms of the interaction, indicate that there are parameter regimes where the thermalization we are interested in may in fact not occur. This would limit the range of allowed values of $\gamma$ and $\lambda$. More specifically, there is a transition in the test particle motion from the expected one of a weakly perturbed harmonic oscillator to an effective double well dynamics as the parameters are varied, as shown in Fig. 1 for the case of the velocity-independent interaction model. The figures show both the initial and final cloud of bath oscillator locations in phase space, which take on the form of Gaussian clouds, and the trajectory of the single target particle over a short $(\approx 20$ cycles $)$ time interval. Panel $($ a) shows the target particle trajectory clearly circulating around the 
origin whereas in case (b) the dynamics is clearly off-center. Changing the initial conditions leads to the center of motion switching to a second location symmetrically located with respect to the origin of the configurational space. To fully understand this phenomenon whose description has been already sketched in the former section, we start from the equations of motion for the test particle and look at the conditions for the equilibria in the two extreme cases of $\lambda \rightarrow 0$ and of $\lambda$ much larger than the confinement size in the trap.

\section{A. Velocity-independent interaction}

We look for fixed points of the Hamilton's equations of motion, by setting $\dot{Q}=0, \dot{P}=0$. The equilibrium location $\left(Q^{*}, P^{*}\right)$ satisfies the conditions

$$
\begin{aligned}
P^{*} & =0 \\
M \Omega^{2} Q^{*} & =-\frac{2 \gamma_{E}}{\lambda^{2}} \sum_{n=1}^{N_{b}} \exp \left[-\left(q_{n}-Q^{*}\right)^{2} / \lambda^{2}\right]\left(q_{n}-Q^{*}\right) .
\end{aligned}
$$

We first deal with the case of small $\lambda$, which mimics point-like interactions such as those associated with the pseudopotential used in the Gross-Pitaevskii equation, effective mean-field, description of a Bose condensate. In this case, $\exp \left(-\left(q_{n}-Q^{*}\right)^{2} / \lambda^{2}\right) \approx \delta\left(q_{n}-Q^{*}\right)$ which, combined with the $q_{n}-Q^{*}$ factor in the righthand side of Eq. (17), implies $Q^{*}=0$. Thus in this limit there is only one fixed point at the origin, $\left(Q^{*}, P^{*}\right)=(0,0)$.

The opposite limit of large $\lambda$ can be treated by replacing the exponential term with the leading terms of its expansion, which leads to

$$
M \Omega^{2} Q^{*}=-\frac{2 \gamma_{E}}{\lambda^{2}} \sum_{n}\left[1-\frac{\left(q_{n}-Q^{*}\right)^{2}}{\lambda^{2}}\right]\left(q_{n}-Q^{*}\right)=\frac{2 \gamma_{E}}{\lambda^{2}}\left(N_{b}-3 N_{q}\right) Q^{*}-\frac{2 \gamma_{E} N_{b}}{\lambda^{4}} Q^{* 3},
$$

where we have used the fact that $\sum_{n} q_{n}^{\alpha}$ is assumed to be nonzero only when $\alpha$ is even, i.e. a symmetric probability density function $q_{n}$, and we have introduced the pure number $N_{q}=\sum_{n=1}^{N_{b}}\left(q_{n} / \lambda\right)^{2}<<N_{b}$ in the large $\lambda$ limit we are considering. The functional form of this relation is $A\left(Q^{*}\right)^{3}=B Q^{*}$ which means that the possible solutions are $Q^{*}=0$ and $Q^{*}= \pm \sqrt{B / A}$ with $A=2 \gamma_{E} N_{b} / \lambda^{4}$ and $B=-M \Omega^{2}+2 \gamma_{E}\left(N_{b}-3 N_{q}\right) / \lambda^{2}$. The threshold for the onset of the $Q^{*} \neq 0$ fixed points is consistent with the analysis following Eq. (10) in the limit of $N_{b}>>N_{q}$, as they both yield a threshold value of $\bar{\gamma}_{E}=M \Omega^{2} \lambda^{2} /\left(2 N_{b}\right)$.

Moreover, a stability analysis shows that above the threshold $\bar{\gamma}_{E}$ the fixed point $Q^{*}=0$ becomes unstable. Given that the fixed point at $(0,0)$ is present both in the small as well as large $\lambda$ limits, a fixed point stability analysis around this phase space location should be sufficient to gauge the transition from effective single to double-well behavior in the test particle dynamics. This requires the introduction of

$$
\begin{aligned}
& Q=Q^{*}+\eta=\eta, \\
& P=P^{*}+\epsilon=\epsilon,
\end{aligned}
$$

leading to the following equations for the small perturbation from the fixed point

$$
\begin{aligned}
& \dot{\eta}=\frac{\epsilon}{M}, \\
& \dot{\epsilon}=\left(-M \Omega^{2}+\frac{2 \gamma_{E} N_{b}}{\lambda^{2}}-\frac{4 \gamma_{E} N_{q}}{\lambda^{2}}\right) \eta .
\end{aligned}
$$

The corresponding eigenvalues become imaginary under the following inequality

$$
\frac{2 \gamma_{E}}{\lambda^{2}}\left(N_{b}-2 N_{q}\right)<M \Omega^{2},
$$

which leads to stable, oscillatory dynamics around the origin. In other words, satisfying the inequality specifies the parameters for a single-well potential. Note that $N_{q}$ has an inverse square dependence on $\lambda$ so the boundary equality involves a quadratic in $\lambda^{2}$. Alternatively, one can numerically find the fixed point value for $Q^{*}$ by turning Eq.(19) into an iterative map. We will discuss this later after first completing the analysis for the velocity-dependent form of the interaction. 

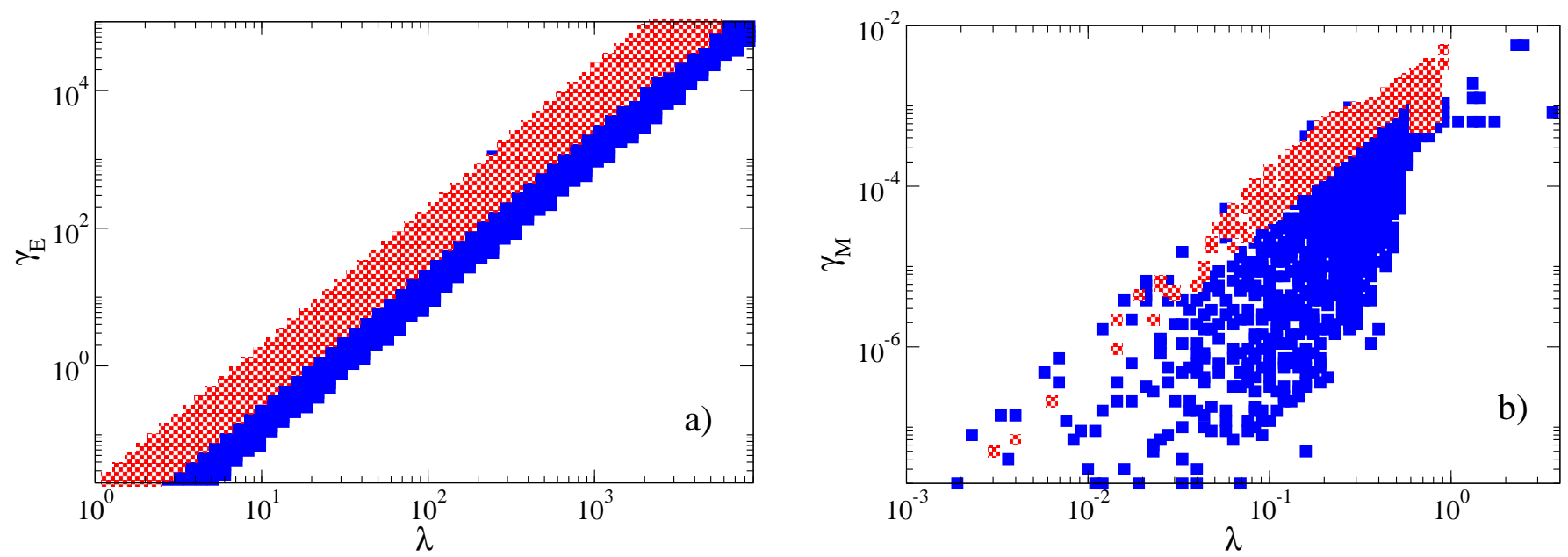

FIG. 2: (Color online) Stability diagrams in parameter space for the dynamical fixed point at $\left(P^{*}, Q^{*}\right)=(0,0)$ for the two forms of the interaction Hamiltonians, velocity-independent (a) $\left(\gamma_{E}, \lambda\right.$ parameter space) and velocity-dependent (b) $\left(\gamma_{M}, \lambda\right.$ parameter space). The white background denotes values where the origin is stable (effective single-well potential). The blue region corresponds to fixed points off the origin (due to the effective double-well dynamics), while the red (chessboard-shaded) region is a boundary where our iterative scheme does not converge to within $\epsilon=10^{-10}$, which may be viewed as a critical boundary. Other relevant parameters in both cases shown are $N_{b}=10^{3}, m=M=1, \omega_{n}=1, \Omega=144 / 89$, and $T_{b}=0.5$. The convergence condition is set by $\epsilon$ and the maximum number of iterations is $10^{4}$. Masses, angular frequencies, temperatures, and parameters $\gamma_{E}, \gamma_{M}$, and $\lambda$ are expressed in arbitrary units.

\section{B. Velocity-dependent interaction}

By repeating analogous considerations for the velocity-dependent case, the equilibrium location $\left(Q^{*}, P^{*}\right)$ can be shown to satisfy the conditions

$$
\begin{aligned}
\frac{P^{*}}{M} & =\frac{2 \gamma_{M}}{M} \sum_{n=1}^{N_{b}}\left(\frac{p_{n}}{m}-\frac{P^{*}}{M}\right) \exp \left[-\left(q_{n}-Q^{*}\right)^{2} / \lambda^{2}\right] \\
M \Omega^{2} Q^{*} & =-\frac{2 \gamma_{M}}{\lambda^{2}} \sum_{n=1}^{N_{b}}\left(\frac{p_{n}}{m}-\frac{P^{*}}{M}\right)^{2}\left(q_{n}-Q^{*}\right) \exp \left[-\left(q_{n}-Q^{*}\right)^{2} / \lambda^{2}\right] .
\end{aligned}
$$

In the case of small $\lambda, Q^{*}=0$ and Eq. (22) reduces to

$$
\frac{P^{*}}{M}=-\frac{2 \gamma_{M}}{M^{2}} P^{*},
$$

modulo a multiplicative constant on the righthand side, which also means $P^{*}=0$. Thus here again the origin in phase space, $(0,0)$, is the only equilibrium point in the case of small $\lambda$.

The opposite limit of large $\lambda$ can be treated again with the expansion of the exponential which leads to

$$
\begin{aligned}
\frac{P^{*}}{M} & =\frac{2 \gamma_{M}}{M} \sum_{n}\left(\frac{p_{n}}{m}-\frac{P^{*}}{M}\right)\left[1-\frac{\left(q_{n}-Q^{*}\right)^{2}}{\lambda^{2}}\right] \\
& =-\frac{2 \gamma_{M} N_{b}}{M^{2}} P^{*}-\frac{2 \gamma_{M}}{M} \sum_{n}\left(\frac{p_{n}}{m}-\frac{P^{*}}{M}\right) \frac{q_{n}^{2}-2 q_{n} Q^{*}+\left(Q^{*}\right)^{2}}{\lambda^{2}} \\
& =-\frac{2 \gamma_{M} N_{b}}{M^{2}} P^{*}+\frac{2 \gamma_{M} C_{2}}{M^{2}} P^{*}+\frac{2 \gamma_{M} C_{3}}{M^{2} \lambda^{2}} P^{*}\left(Q^{*}\right)^{2},
\end{aligned}
$$

where we have used the fact that $\sum_{n} p_{n}^{\alpha} q_{n}^{\beta}$ is assumed to be nonzero only when $\alpha$ and $\beta$ are both even, i.e. a symmetric probability density function in both $p_{n}$ and $q_{n}$, and the constants $c_{i}$ implicitly defined above are largely unimportant for our qualitative considerations. What is clear from the last step is that $P^{*}=0$ is still the equilibrium momentum 
value, and this implies conditions on $Q^{*}$

$$
\begin{aligned}
M \Omega^{2} Q^{*} & =-\frac{2 \gamma_{M}}{\lambda^{2}} \sum_{n} \frac{p_{n}^{2}}{m^{2}}\left[1-\frac{\left(q_{n}-Q^{*}\right)^{2}}{\lambda^{2}}\right]\left(q_{n}-Q^{*}\right) \\
& =-\frac{2 \gamma_{M}}{\lambda^{2}} \sum_{n} \frac{p_{n}^{2}}{m^{2}}\left(q_{n}-Q^{*}\right)+\frac{2 \gamma_{M}}{\lambda^{4}} \sum_{n} \frac{p_{n}^{2}}{m^{2}}\left(q_{n}-Q^{*}\right)^{3} \\
& =+\frac{2 \gamma_{M} d_{1}}{\lambda^{2} m^{2}} Q^{*}-\frac{6 \gamma_{M} d_{2}}{\lambda^{4} m^{2}} Q^{*}-\frac{2 \gamma_{M} d_{1}}{\lambda^{4} m^{2}}\left(Q^{*}\right)^{3},
\end{aligned}
$$

where $d_{1}=\sum_{n} p_{n}^{2}$ and $d_{2}=\sum_{n} p_{n}^{2} q_{n}^{2}$. The functional form of this relation is $A\left(Q^{*}\right)^{3}=B Q^{*}$ which means that the possible solutions are $Q^{*}=0$ and $Q^{*}= \pm \sqrt{B / A}$.

Since even in this case $(0,0)$ is always a fixed point, a stability analysis around this phase space location analogous to the previous case should be sufficient to identify the threshold for bistable behavior, obtaining the linearized relations

$$
\begin{aligned}
\dot{\eta} & =\left(\frac{1}{M}+\frac{2 \gamma_{M} c_{0}}{M^{2}}\right) \epsilon \equiv b \epsilon, \\
\dot{\epsilon} & =\left(-M \Omega^{2}+\frac{2 \gamma_{M} c_{p}}{m^{2} \lambda^{2}}-\frac{4 \gamma_{M} c_{q p}}{m^{2} \lambda^{2}}\right) \eta \equiv a \eta,
\end{aligned}
$$

where

$$
c_{0}=\sum_{n=1}^{N_{b}} \exp \left(-q_{n}^{2} / \lambda^{2}\right), \quad c_{p}=\sum_{n=1}^{N_{b}} p_{n}^{2} \exp \left(-q_{n}^{2} / \lambda^{2}\right), \quad c_{q p}=\sum_{n=1}^{N_{b}} p_{n}^{2}\left(q_{n} / \lambda\right)^{2} \exp \left(-q_{n}^{2} / \lambda^{2}\right) .
$$

The eigenvalues $\Lambda$ of the Jacobian matrix satisfy the condition $\Lambda^{2}=a b$ which given that $b>0$ (for $\gamma_{M}>0$ ) results in $a<0$ in order for the eigenvalues to be imaginary indicating stability. This leads to

$$
\frac{2 \gamma_{M}}{m^{2} \lambda^{2}}\left(c_{p}-2 c_{q p}\right)<M \Omega^{2}
$$

The other case, for fixed $\gamma_{M}$, is more involved. Note that the both terms on the left of the inequality vary with time, with the evolution of the bath variables, though each can be replaced by the averages over the bath multiplied by $N_{b}$.

As indicated earlier, away from these limiting cases, we can use an iterative scheme to numerically determine the location of the fixed point. This is made easier by the fact that $P^{*}=0$ for both forms of the interaction and only $Q^{*}$ has to be determined. A wide range of parameter values can be considered and for each pair of $\left(\lambda, \gamma_{E}\right)$ or $\left(\lambda, \gamma_{M}\right)$ values, the location of the fixed point numerically computed. As depicted in Fig2(a) and Fig2(b) ,for velocityindependent and velocity-dependent cases respectively, the parameter values where the origin is a globally stable fixed point can be distinguished from those where the fixed point moves away. These phase diagrams can prove useful in determining regimes where the relaxation between the test particles and bath occurs more readily. It is worth noting the existence of a critical boundary layer where convergence slows down considerably, denoted by the red points in both panels in Fig. 2. The precise implications of this region is still to be determined though we suspect that this may simply be the consequence of the merger of the two wells (of the double-well potential) in becoming a single well.

\section{THERMALIZATION DYNAMICS: NUMERICAL RESULTS}

Having gained some insight into the behavior of a single target particle interacting with the oscillators in the bath, we now move to the issue of thermalization. In our numerical exploration, we considered the distinct thermal relaxation scenarios of a single target oscillator interacting with the $N_{b}$ bath oscillators as well the more realistic situation where $N_{p}$ target oscillators interact with the bath but the results presented here focus on the latter case. The equations of motion were integrated using a variable step algorithm which preserved the Hamiltonian to machine precision. When multiple target oscillators were considered, the initial conditions were drawn from a thermal distribution with temperature $T_{p}$. Again, with an eye on the sympathetic cooling application, the temperature of the target particles was chosen to be higher than that of the bath, that is $T_{p}>T_{b}$. In other words, the species to be cooled are the target particles with the coolant species making up the bath. All the oscillators in each category were assigned the same angular frequency, $\omega$ for bath oscillators and $\Omega$ for the target ones. In light of earlier analysis within the CaldeiraLeggett framework [5] and the conditions for sympathetic cooling of mixtures, we consider $\omega \neq \Omega$. Our choice of 

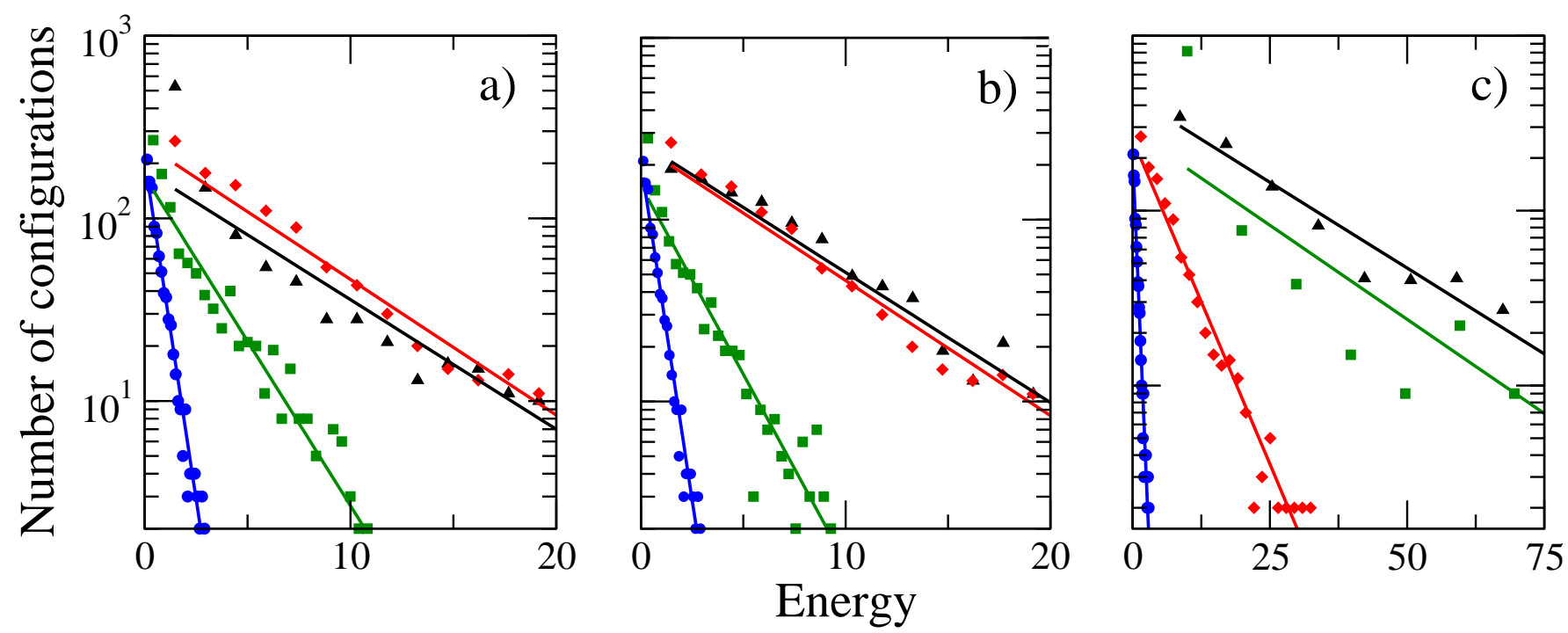

FIG. 3: (Color online) Energy spectrum of initial target system (red, diamond), bath (blue, circle) and final target (black, triangle), bath (green, square) for (a),(b) velocity independent and (c) velocity dependent interactions. In each instance, $N_{b}=N_{p}=10^{3}$ and initial temperatures set in the numerical code equal to $T_{b}=0.5$ for the bath, and $T_{p}=5$ for the target system (corresponding to inverse temperatures $\beta_{b}=T_{b}^{-1}=2$ and $\beta_{p}=T_{p}^{-1}=0.2$, respectively), $\omega=1.0, \Omega=144 / 89$. The parameters of the interacting Hamiltonian and the running time of the simulations are (a) $\gamma_{E}=0.1, \lambda=0.01, t_{\mathrm{run}}=5 \times 10^{4}$, (b) $\gamma_{E}=0.1, \lambda=0.1, t_{\text {run }}=2 \times 10^{4}$, and (c) $\gamma_{M}=0.1, \lambda=0.1, t_{\text {run }}=10^{4}$. The unweighted best fit to the Boltzmann distributions, shown as continuous lines around the points of each curve, provides $\beta_{b}^{(i)}=1.71 \pm 0.06$ and $\beta_{t}^{(i)}=0.171 \pm 0.009$ for the actual initial bath and target system inverse temperatures, respectively, and (a) $\beta_{b}^{(f)}=0.41 \pm 0.03, \beta_{p}^{(f)}=0.16 \pm 0.01$, (b) $\beta_{b}^{(f)}=0.48 \pm 0.03, \beta_{p}^{(f)}=0.165 \pm 0.007$, (c) $\beta_{b}^{(f)}=0.049 \pm 0.009, \beta_{p}^{(f)}=0.045 \pm 0.003$, for the final temperatures. Masses, angular frequencies, temperatures, parameters $\gamma_{E}, \gamma_{M}, \lambda$, energies and inverse temperatures are expressed in arbitrary units.

$\Omega=144 / 89$ is intended to preclude any low-order resonances between bath and target particles though, as discussed a little later, this should not be a problem. The two other parameters of relevance are the range of the interaction $\lambda$ and their interaction strengths $\gamma_{E}$ (velocity-independent) and $\gamma_{M}$ (velocity-dependent). Although we numerically explored a range of model parameters, we have decided to limit the results shown to some illustrative cases which highlight new phenomena as well as being pertinent to application to atomic mixtures. One point to note is that we sample the dynamics every $\Delta t=0.01$ and our units of time are simply the number of time steps. This also means that a single period of a bath oscillator is about 628 time steps, which should be treated simply as a reference rather than a relevant timescale. We will return to this issue later when discussing our results.

Since we are primarily interested in the thermalization effect, all the cases discussed from now on involve multiple, $N_{p}$, target particles interacting with $N_{b}$ bath oscillators. Here again, we considered both velocity-independent and velocity-dependent forms of the interaction though there were very few qualitative differences between the behavior seen in the two instances. The initial conditions for both subsystems are drawn from thermal distributions and we considered a range of interaction times. The interaction parameters $\lambda, \gamma_{E}$ and $\lambda, \gamma_{M}$ have been chosen to correspond to situations where the fixed point at the origin in phase space is stable. The nonlinear regime discussed in Sections II and III would suggest a more favorable thermalization dynamics in the nonlinear regime when the fixed points are away from the origin in phase space. However, the lack of overlap in the configuration space and the local form of the interaction makes this regime uninteresting for the task of a fast thermalization. The energy or spectral distribution of each subsystem was then considered to extract the final temperature (actually inverse temperature) of both target particles and bath oscillators. It should be noted here that our use of the term 'temperature' is, in general, inexact given the dynamical and, hence, inherently non-equilibrium nature of the problem. Nevertheless, the slope obtained from fitting the logarithm of the energy distribution can be thought of as the effective temperature at a given time, with the error in its determination assessing also the effectiveness of the description in terms of this parameter alone. At any given time, we consider the total energy of the test particles, the total energy of the bath particles, and their 

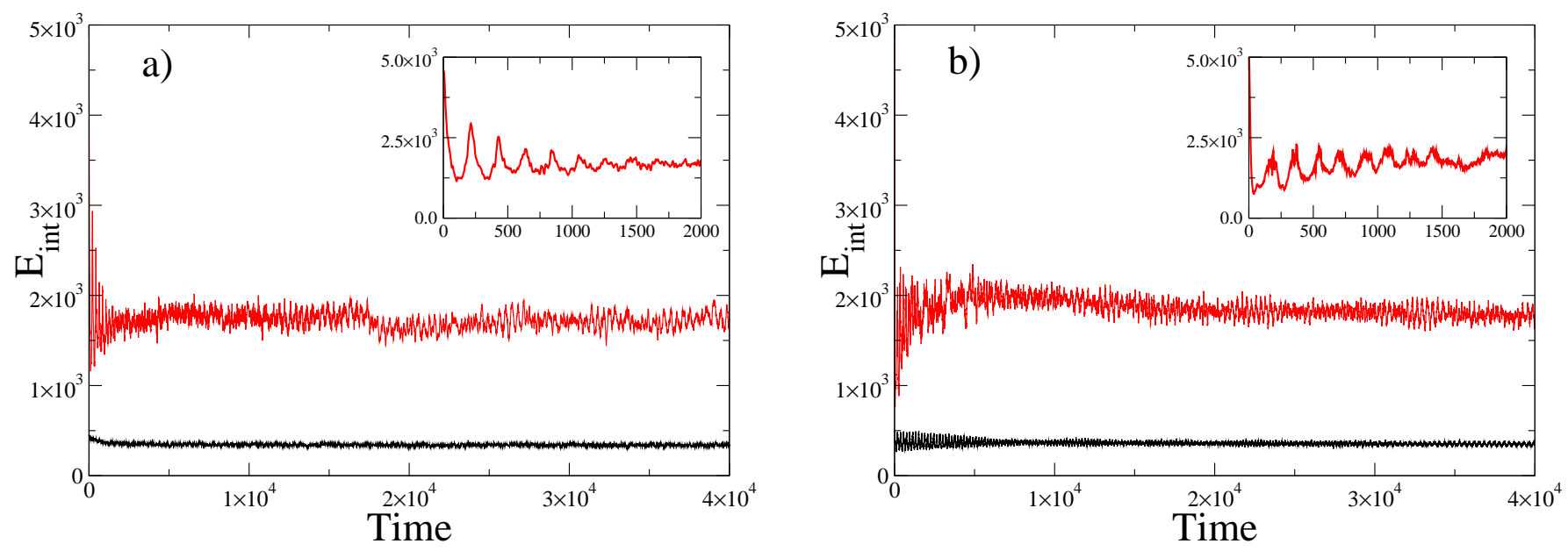

FIG. 4: (Color online) Interaction energy as a function of time for velocity independent (a) and velocity dependent (b) interactions. The plots are for two choices of $\lambda=0.01$ (black, bottom curve) and $\lambda=0.1$ (red, top curve), with $\gamma_{E}=0.1$ (a) and $\gamma_{M}=0.02$ (b), where the latter parameter was intentionally chosen to have comparable final interaction energy in the two situations. Oscillations which appear on short timescales are shown in the insets. All other parameters are chosen as in Fig. 3, and both time (in steps) and energies are expressed in arbitrary units.

interaction energy, defined as

$$
\begin{aligned}
E_{\mathrm{p}}(t) & =\sum_{m=1}^{N_{p}} E_{m, \mathrm{p}}(t)=\sum_{m=1}^{N_{p}}\left(\frac{P_{m}^{2}(t)}{2 M}+\frac{1}{2} M \Omega^{2} Q_{m}^{2}(t)\right), \\
E_{\mathrm{b}}(t) & =\sum_{n=1}^{N_{b}} E_{n, \mathrm{~b}}(t)=\sum_{n=1}^{N_{b}}\left(\frac{p_{n}^{2}(t)}{2 m}+\frac{1}{2} m \omega^{2} q_{n}^{2}(t)\right), \\
E_{\text {int }}(t) & =\sum_{m=1}^{N_{p}} \sum_{n=1}^{N_{b}} H_{\mathrm{int}}\left(Q_{m}(t), P_{m}(t), q_{n}(t), p_{n}(t)\right) .
\end{aligned}
$$

The distribution of energies for test and bath particles after a fixed interaction time $t_{\text {run }}$, that is the histograms of $E_{m, \mathrm{p}}\left(t_{\text {run }}\right)$ and $E_{n, \mathrm{~b}}\left(t_{\text {run }}\right)$ respectively, can then be used to extract the effective inverse temperatures for the two subsystems. As we will discuss later, the energy content in the interaction also appears to play an important role in determining the final temperatures in both our models.

Figure 3 shows the results of this construction for some illustrative cases where the lines drawn are least squares fits to the points which display the energy distributions at the times specified in the captions. In each case, both initial and final distributions of the two subsystems, particle and bath, are shown. The slope of the fit provides the corresponding $\beta=1 /\left(k_{B} T\right)$ at time $t_{\text {run }}$. Given the poor statistics at the higher ends of the energy spectra, we have also implemented weighted least squares fits which appear to be more accurate for any quantitative analysis though the qualitative inferences are rather robust with respect to the data fitting. The results in Fig. 3 readily suggest the role the effective number of bath oscillators interacting with each target particle, $N_{\lambda}$, plays in promoting

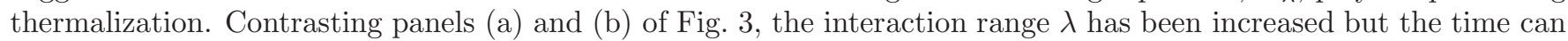
then been decreased to get the effective temperatures to be the same. Plot (c) in Fig. 3 shows the effect of the velocity dependent interaction at shorter times, and implies temperature oscillations in the early stage of thermalization. The oscillatory phenomenon is more clearly seen by considering the time dependence of the interaction energy, defined as $E_{\text {int }}=\gamma_{E} \sum_{n=1}^{N_{b}} \sum_{m=1}^{N_{p}} \exp \left[-\left(q_{n}-Q_{m}\right)^{2} / \lambda^{2}\right]$ in the case of velocity-independent interactions. As shown in the panels of Fig. 4 , for both interaction types, there is a sharp decrease in this measure to essentially a quasi-stationary value, with the inset emphasizing the energy oscillations at early times. The velocity-dependent case shows more quasi-periodicity than in the velocity-independent situation.

The observant reader will also notice that in Fig. 3(c) the final inverse temperatures of both bath and target subsystems are lower than the initial values, implying heating of both species. To explore the dynamics of thermalization more clearly, and in particular this double heating effect, we consider the time dependence of the effective temperatures of bath and target subsystems. Based on what we deduced earlier, thermalization should occur faster in the case with 

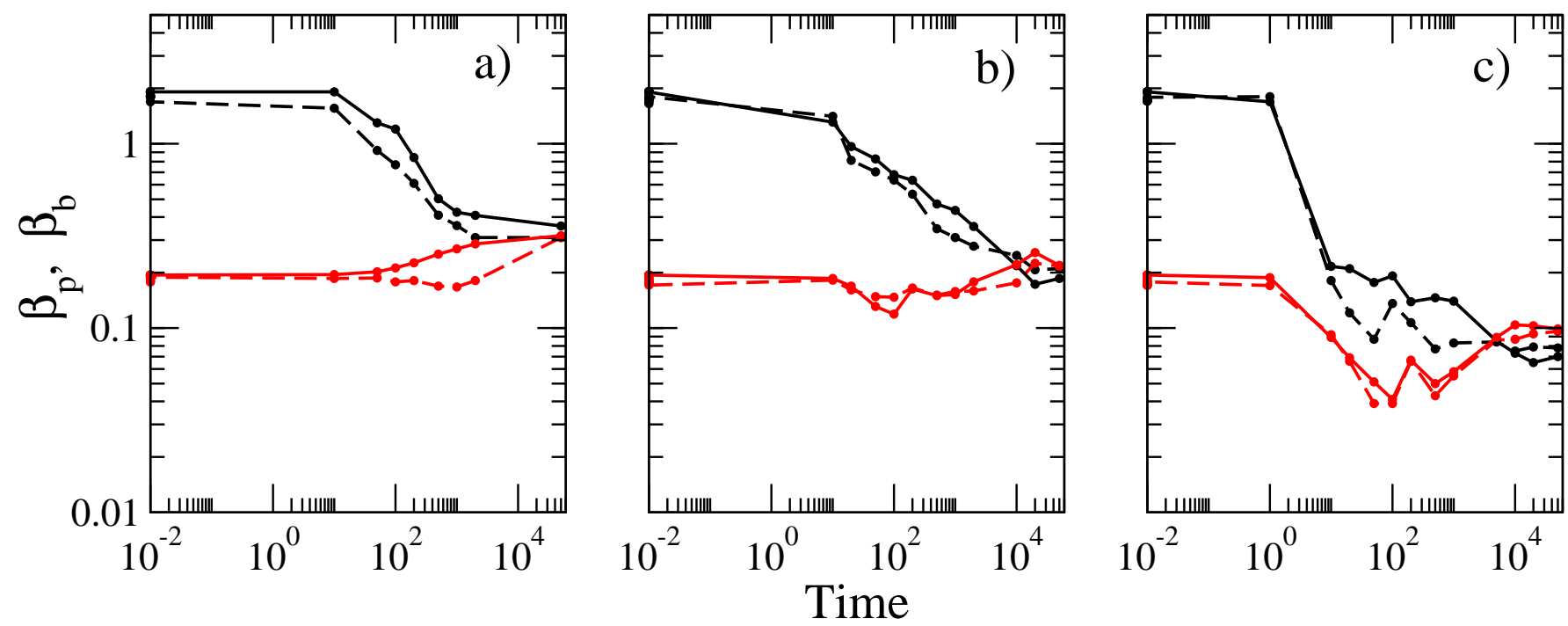

FIG. 5: (Color online) Relaxation dynamics for target and bath subsystems for velocity independent interactions. The effective inverse temperature is shown versus time for the test particles with unweighted (red dashed line) and weighted (red continuous line) fitting to a Boltzmann distribution, bottom curves on the left side of each plot, and for the bath particles with unweighted (black dashed line) and weighted (back continuous line) fitting, top curves on the left side of each plot. In each instance, $N_{b}=N_{p}=10^{3}, T_{b}=0.5, T_{p}=5.0, \omega=1.0, \Omega=144 / 89$, with interaction parameters $(\mathrm{a}) \gamma_{E}=0.1, \lambda=0.01 ;(\mathrm{b})$ $\gamma_{E}=0.1, \lambda=0.1$, and (c) $\gamma_{E}=0.5, \lambda=0.1$. While the weighted fits are expected to be more accurate, significant deviations from the results of unweighted fits can be used to infer the presence of strong deviations from a Boltzmann distribution. The spread in the left most data points is a consequence of small statistics associated with the sampling of the thermal initial conditions through different seeds for the random number generator. The typical relative errors associated with the inverse temperature determination through the fits are about 10\%, and masses, angular frequencies, parameters $\gamma_{E}, \lambda$, energies, and inverse temperatures are expressed in arbitrary units.

larger $\lambda$, irrespective of the nature of the interaction, and relatively quicker for the velocity-dependent interaction. The latter expectation is trickier to show numerically as equivalent parameter regimes for the two cases are hard to determine. This is readily seen from the parameter phase diagrams shown earlier. However, as seen from contrasting Figs. 5 and 6, where three cases for each interaction type is shown, the qualititative features are very similar. In each figure, what is shown is (a) where thermalization does not occur even at the longest time considered; (b) where there are clear signs that thermalization is occurring and (c) an anomalous situation where the final temperatures for both bath and target are higher than their initial values. Figures 5(a) and (b) (and their equivalents in Fig. 6) clearly show that the temporal scale for relaxation decreases on increasing the spatial region of interaction (increasing $\lambda$ ). This in turn implies faster equilibration due to the increase in the average number of interacting particles at any given time.

We now turn to the anomalous situation seen in both Figs. 5(c) and 6(c). This occurs, for certain initial configurations, when a large amount of interaction energy is initially present in the system, typically in the large $\gamma_{E}$ or $\gamma_{M}$ limit where we will have $E_{\text {int }}>>E_{b}, E_{p}$. This is a distinctive feature of our approach, as usually any initial interaction energy or initial correlation between two subsystems is assumed to be nearly negligible with respect to the internal energy of the subsystems. This last situation is however characteristic of solid state systems, in which the contact between two bodies is limited to their surfaces, and it is therefore marginal with respect to the internal energy of the bodies. Here instead the two 'bodies' are penetrating, as atoms of the two different species see each other in the whole available trapping volume. Clearly this is outside the usual view of systems in thermal contact where the interface plays no role in the thermalization process. Here it does and, given the excess energy content, can heat both the systems as if there is a fictitious third, hotter subsystem in the problem, or if the interaction energy acts as a sort of 'latent heat' released during the time evolution. Of course this initial interaction energy can also be minimized, even at a relatively large value of $\gamma_{E}$ or $\gamma_{M}$, by tactically choosing the initial conditions in such a way that the oscillators of the two systems are very close to each other (in the case of attractive interactions) or very far apart (in the case of repulsive interactions). Likewise, the sudden change in the interaction between the two subsystems, for instance by exploiting Feshbach resonances of the interspecies scattering length, allows for implosions or explosions of the atomic clouds, as in the 'Bosenova' effect 25 27]. All these situations are in principle covered by our simulation technique by properly choosing initial conditions for the bath particles and/or time-dependent interactions couplings.

Regarding the thermalization timescale and its dependence upon the parameters $\gamma_{E}, \gamma_{M}$, and $\lambda$, the plots in Figs. 5 

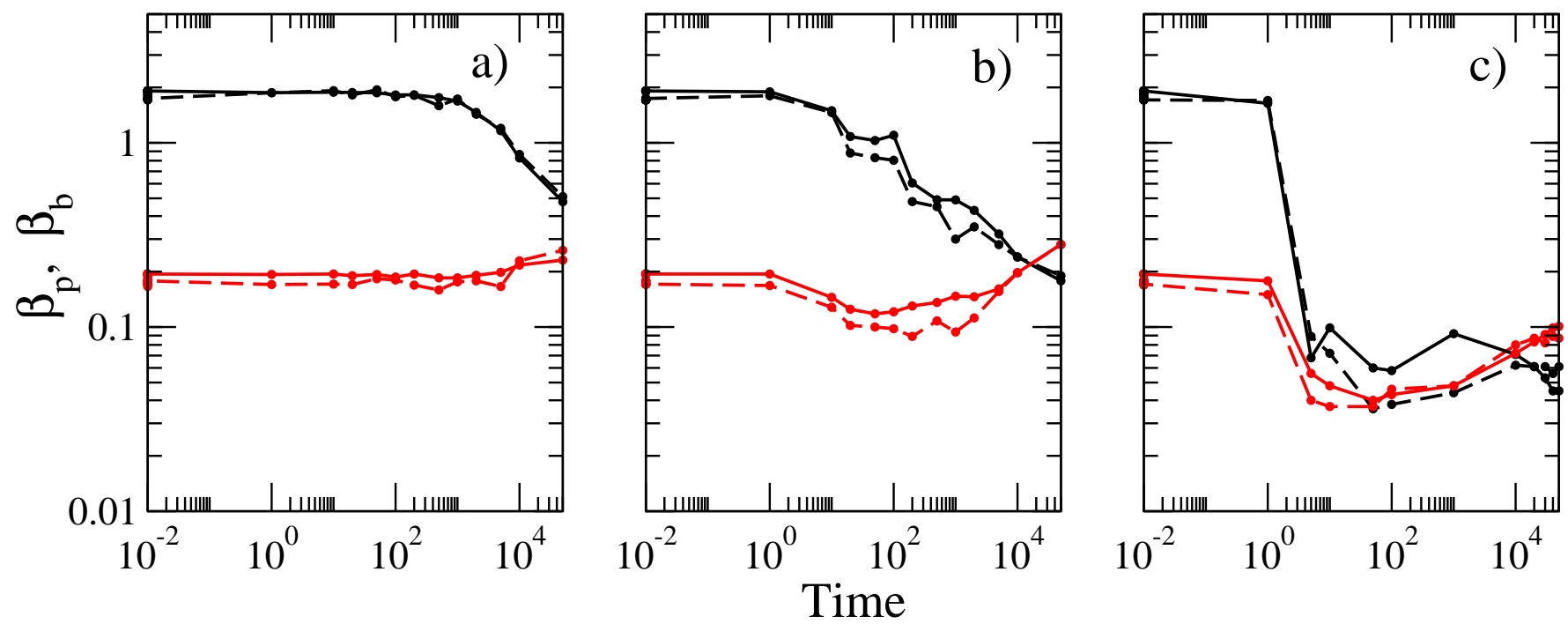

FIG. 6: (Color online) Relaxation dynamics for target and bath subsystems for velocity dependent interactions. The effective inverse temperature is shown versus time for the test particles with unweighted (red dashed line) and weighted (red continuous line) fitting to a Boltzmann distribution, bottom curves on the left side of each plot, and for the bath particles with unweighted (black dashed line) and weighted (black continuous line) fitting, top curves on the left side of each plot. Like in Fig. 5, we have $N_{b}=N_{p}=10^{3}, T_{b}=0.5, T_{p}=5.0, \omega=1.0, \Omega=144 / 89$, while the interaction parameters are $(\mathrm{a}) \gamma_{M}=0.02, \lambda=0.01$; (b) $\gamma_{M}=0.02, \lambda=0.1$ and (c) $\gamma_{M}=0.1, \lambda=0.1$. The spread in the left most data points is a consequence of small statistics associated with the sampling of the thermal initial conditions through different seeds for the random number generator. The typical relative errors associated with the inverse temperature determination through the fits are about $10 \%$, and masses, angular frequencies, parameters $\gamma_{M}, \lambda$, energies, and inverse temperatures are expressed in arbitrary units.

and 6 show that the thermalization time is inversely proportional to the interaction strength as intuitively expected. This can be confirmed by a semiquantitative analysis at least in the simpler case of velocity independent interactions. By visual inspection of the right hand side of Eq. (7), the two relevant timescales for the test particle are the intrinsic one of its free oscillation (the period of oscillation $T=2 \pi / \Omega$ ) and a response time to the force due to any single oscillator of the bath, $\tau_{r} \simeq 2 \pi\left[M \lambda^{2} /\left(2 \gamma_{E}\right)\right]^{1 / 2}$. Thus, the thermalization timescale, proportional to $\tau_{r}$ varies with the coupling strength as $\propto 1 / \sqrt{\gamma_{E}}$. Indeed, in Fig. [5(b) and (c), corresponding to the same $\lambda$ with coupling strengths $\gamma_{E}$ differing by a factor 5 , the crossing between the two curves corresponding to the weighted fits for the inverse temperature occurs at times in the approximate ratio of $2\left(\simeq 10^{4}\right.$ time steps for the case in $(\mathrm{b}), \simeq 5 \times 10^{3}$ times steps in (c)). A similar analysis is unfortunately not possible for the dependence of the thermalization time upon the interaction range $\lambda$. A naive extension of the argument above indicates a response time increasing linearly with $\lambda$, which defies physical intuition as in this case more and more oscillators interact with the test particle. This, in fact, is precisely the issue as $\tau_{r}$ introduced above does not take into account the number of interacting oscillators from the bath, i.e. the sum term in Eq. (7) which also grows with $\lambda$. Numerically, Fig. [5 (a) and (b) show that increasing $\lambda$ speeds up thermalization. Due to the complicated structure of the Hamilton equations for the velocitydependent case, the above analysis cannot be repeated, however the results shown in Fig. 6 confirms the same trends in this case. Also, notice that the onset of a quasi-stationary regime for the interaction energy in Fig. (4 appears to coincide with the thermalization timescales in Figs. 5] and 6. suggesting that the former may act as a shortcut to study the relaxation to thermal equilibrium or at least to steady states. Finally, we show in Fig. 7 the long time behavior in the velocity-independent case, both for the evolution of the inverse temperatures (left panel) as well as the interaction energy (right panel). We note that not only do the timescales for thermalization at very short times coincide for these two observables, corroborating the remark made in the previous sentence, but also that their longtime behavior seem to exhibit fluctuations of similar relative amplitude. Further, Fig. 7(a) confirms that the crossing of the inverse temperatures clearly visible at shorter times (in both Figs. 5(b) and (c)) is a temporary feature, leading to a stabilization of the temperatures for later times. 

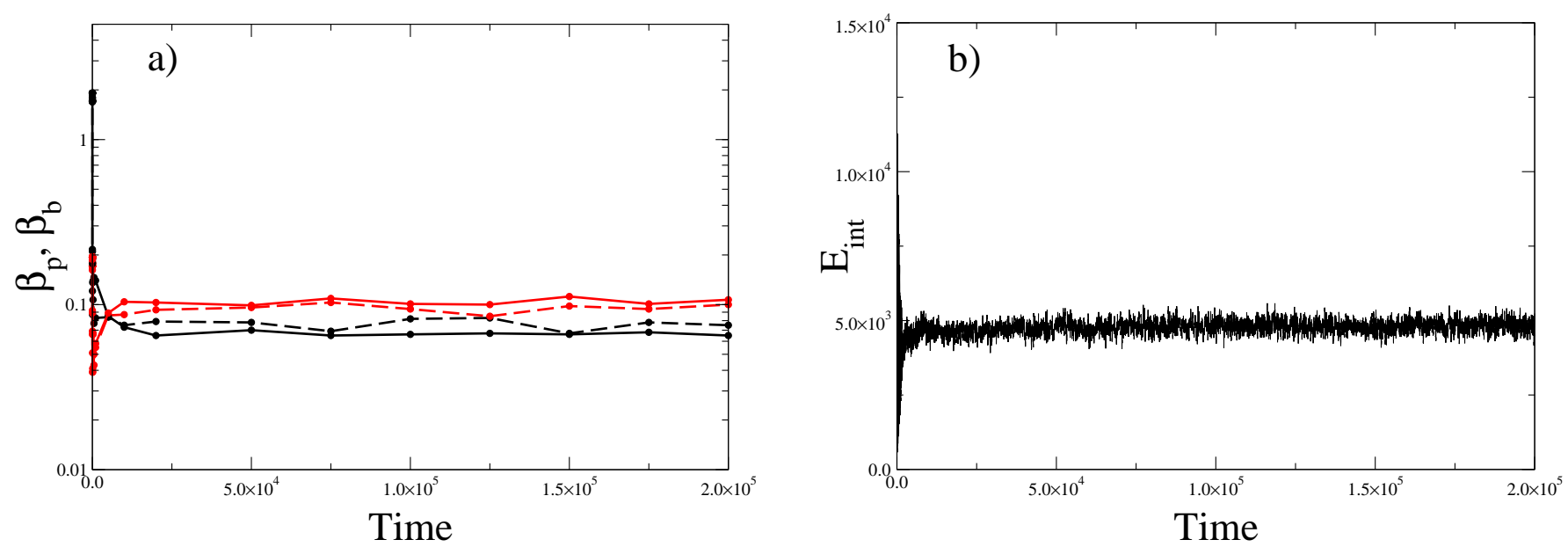

FIG. 7: (Color online) Long time simulation for the velocity-independent case. On the left plot (a) the effective inverse temperature is shown versus time for the test particles with unweighted (red dashed line) and weighted (red continuous line) fitting to a Boltzmann distribution, as in the two top curves on the right side of the plot, and for the bath particles with unweighted (black dashed line) and weighted (black continuous line) fitting, as in the two bottom curves on the right side of the plot. On the right plot (b) the corresponding interaction energy as a function of time is depicted. The parameters are chosen as in the case (c) of Fig. 5, and all quantities are expressed in arbitrary units.

\section{CONCLUSIONS}

In conclusion, we have analyzed the thermalization dynamics of atoms trapped in a confining potential under the action of another gas with a generic temperature and different trapping frequency. The analysis has been carried out through molecular dynamics simulations adapting the Caldeira-Leggett model to the specific context of atoms oscillating at well-defined frequencies in a harmonic trap. The nonlinear form of the interaction between the two atomic species required an assessment of the dynamical stability which limits the range of the parameters of the model for enabling efficient thermalization. Our numerical analysis does not rely on the weak-coupling assumption typically underlying the application of the Caldeira-Leggett model to open systems. A manifest implication of this more generalized framework is the fact that in the strong coupling regime the very definition of Boltzmann equilibrium distribution is at stake, and then only effective temperatures can be defined. This is in line with former analyses in the context of chemical physics, as in 28, 29] in which the preservation of the Boltzmann distribution is ensured only in a weak interaction regime, and therefore fails for strongly exothermic chemical reactions (for a discussion of the strong coupling regime in open quantum systems, with similar outcomes in terms of lack of thermalization, see [30]).

The simulations have been performed for a purely classical setting which, in terms of atomic clouds, seem justified whenever the sample temperature $T$ fulfils the condition $T>>\hbar \omega / k_{B}$, as usual in atomic trapping even at the lowest explored temperatures. Quantum effects will however influence the thermalization dynamics at finite temperature through the deviations from the classical Dulong-Petit specific heat law, which could be incorporated in the molecular dynamics code [31]. In particular, this affects the dynamics of thermalization for mixtures of Bose and Fermi gases as their thermal response is quite different, with the specific heat scaling differently with temperature as they become degenerate. Simulations in this setting should be able to confirm the existence of a heat capacity mismatch that strongly hinders the capability of a Bose gas to sympathetically cool a Fermi gas, as discussed in 32 35]. If the interaction strength between the two species is not large enough, the Fermi gas will not reach an equilibrium state with the Bose gas undergoing evaporative cooling, an issue of crucial relevance for precision thermometry of degenerate Fermi gases as emphasized in [15]. It is also worth remarking that, in the case of harmonic oscillators, classical dynamics and quantum dynamics of the centroid coincide, based on the Ehrenfest theorem, so there is decoupling between average motion and quantum fluctuations. This allows the analysis, even in a quantum framework, of the simpler case of a classical harmonic oscillator, with additional but separate considerations for the fluctuation part of its dynamics in its quantum counterpart, provided that the nonlinear interaction term is perturbative with respect to the uncoupled dynamics.

Various aspects of our model could be expanded in future work. Specifically, the role of the particle masses of the two systems in determining thermal equilibrium. This could allow the generalization of the notion of Rayleigh and Lorentz gases, corresponding to the two extreme limits of $m<<M$ and $m>>M$ respectively, to arbitrary 
interaction potentials, not just restricted to the case of hard-sphere interactions discussed in Ref. [36]. The two phenomenological parameters of the interaction need to be mapped to ab initio parameters of interatomic interactions in order to promote quantitative analyses for concrete experimental settings. Further, by changing our choice of initial conditions, situations in which fast atoms with a narrow Gaussian energy distribution approach an equilibrium thermal cloud can also be recovered, bridging the experimental studies performed in [37]. The model could be also be applied to more general dynamical situations, for instance time-dependent interactions in an atomic mixture resulting from a sudden change of the interspecies scattering length or sympathetic cooling under targeted driving of the trapping frequencies, as in shortcuts to adiabaticity 38 -40]. The latter situation could make more stringent the practical feasibility of the proposal introduced in Ref. [41], or, more generally, the optimization of a targeted quantum state at finite temperature discussed in Ref. [42].

[1] V. B. Magalinskii, Sov. Phys. JETP 9, 1381 (1959).

[2] P. Ullersma, Physica 52, 27 (1966); ibid. 32, 74 (1966); ibid. 32, 90 (1966).

[3] A. O. Caldeira and A. J. Leggett, Phys. Rev. Lett. 46, 211 (1981).

[4] A. O. Caldeira and A. J. Leggett, Ann. Phys. 149, 374 (1983).

[5] S. T. Smith and R. Onofrio, Eur. Phys. J. B 61, 271 (2008).

[6] P. Hanggi, G.-L. Ingold, and P. Talkner, New J. Phys. 10, 115008 (2008).

[7] H. Hasegawa, Phys. Rev. E 83, 021104 (2011).

[8] A. Carcaterra and A. Akay, Phys. Rev. E 84, 011121 (2011).

[9] H. Hasegawa, J. Math. Phys. 52, 123301 (2011).

[10] H. Hasegawa, Phys. Rev. E 84, 011145 (2011).

[11] J. R. Ensher, D. S. Jin, M. R. Matthews, C. E. Wieman, and E. A. Cornell, Phys. Rev. Lett. 77, 4984 (1996).

[12] B. DeMarco and D. S. Jin, Science 285, 1703 (1999).

[13] M. Crescimanno, C. G. Kaoy, and R. Peterson, Phys. Rev. A 61, 053602 (2000).

[14] M. J. Holland, B. DeMarco, and D. S. Jin, Phys. Rev. A 61, 053610 (2000).

[15] D. C. McKay and B. DeMarco, Rep. Prog. Phys. 74, 054401 (2011).

[16] S. Lepri, R. Livi, and A. Politi, Phys. Rep. 377, 1 (2003).

[17] R. Onofrio and C. Presilla, Phys. Rev. Lett. 89, 100401 (2002).

[18] R. Onofrio and C. Presilla, J. Stat. Phys. 115, 57 (2004).

[19] M. A. Brown-Hayes and R. Onofrio, Phys. Rev. A 70, 063614 (2004).

[20] J. Catani, G. Barontini, G. Lamporesi, F. Rabatti, G. Thalhammer, F. Minardi, S. Stringari, and M. Inguscio, Phys. Rev. Lett. 103, 140401 (2009).

[21] S. Tassy, N. Nemitz, F. Baumer, C. Höhl, A. Batär, and A. Görlitz, J. Phys. B 43, 4 (2010).

[22] F. Baumer, F. Münchow, A. Görlitz, S. E. Maxwell, P. S. Julienne, and E. Tiesinga, Phys. Rev. A 83, 040702(R) (2011).

[23] V. D. Vaidya, J. Tiamsuphat, S. L. Rolston, and J. V. Porto, Phys. Rev. A 92, 043604 (2015).

[24] A. Konetchnyi, M. B. Mensky, and V. Namiot, Phys. Lett. A 177, 283 (1993).

[25] H. Saito and M. Ueda, Phys. Rev. Lett. 86, 1406 (2001).

[26] E. A. Donley, N. R. Claussen, S. L. Cornish, J. L. Roberts, E. A. Cornell, and C. E. Wieman, Nature 412, 295 (2011).

[27] P. A. Altin, G. R. Dennis, G. D. McDonald, D. Döring, J. E. Debs, J. D. Close, C. M. Savage, and N. P. Robins, Phys. Rev. A 84, 033632 (2011).

[28] E. W. Montroll and K. E. Shuler, J. Chem. Phys. 26, 454 (1957).

[29] H. C. Andersen, I. Oppenheim, K. E. Shuler, and G. H. Weiss, J. Math. Phys. 5, 522 (1964).

[30] Y. Subaşi, C. H. Fleming, J. M. Taylor, and B. L. Hu, Phys. Rev. E 86, 061132 (2012).

[31] H. Dammak, Y. Chalopin, M. Laroche, M. Hayoun, and J.-J. Greffet, Phys. Rev. Lett. 103, 190601 (2009).

[32] A. G. Truscott, K. E. Strecker, W. I. McAlexander, G. B. Partridge, and R. G. Hulet, Science 291, 2570 (2001).

[33] M. Wouters, J. Tempere, and J. T. Devreese, Phys. Rev. A 66, 043414 (2002).

[34] C. Presilla and R. Onofrio, Phys. Rev. Lett. 90, 030404 (2003).

[35] M. Brown-Hayes, Q. Wei, C. Presilla, and R. Onofrio, Phys. Rev. A 78, 013617 (2008).

[36] K. Andersen and K. E. Shuler, J. Chem. Phys. 40, 633 (1964).

[37] P. Zhang, V. Kharchenko, A. Dalgarno, Y. Matsumi, T. Nakayama, and K. Takahashi, Phys. Rev. Lett. 100, 103001 (2008).

[38] X. Chen, A. Ruschhaupt, S. Schmidt, A. del Campo, D. Guéry-Odelin, and J. G. Muga, Phys. Rev. Lett. 104, 063002 (2010).

[39] J.-F. Schaff, P. Capuzzi, G. Labeyrie, and P. Vignolo, New J. Phys. 13, 113017 (2011).

[40] E. Torrontegui, S. Ibáñez, S. Martínez-Garaot, M. Modugno, A. del Campo, D. Guéry-Odelin, A. Ruschhaupt, X. Chen, and J. G. Muga, Adv. At. Mol. Opt. Phys. 62, 117 (2013).

[41] S. Choi, R. Onofrio, and B. Sundaram, Phys. Rev. A 84, 051601(R) (2011).

[42] V. Mukherjee, A. Carlini, A. Mari, T. Caneva, S. Montangero, T. Calarco, R. Fazio, and V. Giovannetti, Phys. Rev. A 88, 062326 (2013). 